\title{
ELECTRONIC PAGES
}

\section{eHEART: www.heartinl.com}

The

he full text of this electronic only article is published on the Heart website-www.heartjnl.com/supplemental

The Coronary Artery Revascularisation in Diabetes (CARDia) trial: a prospective, randomised comparison of optimal coronary angioplasty with use of stenting and abciximab recommended versus up to date coronary artery bypass grafting in patients with diabetes mellitus suitable for either intervention

Kapur A, Malik IS, Bagger JP, Anderson JR, Beatt KJ, Hall RJC, for the CARDia Investigators

Background: Patients with diabetes mellitus have an increased incidence and severity of ischaemic heart disease, which leads to an increased requirement for coronary revascularisation. Evidence to decide on best operative strategy-coronary artery bypass surgery (CABG) or percutaneous coronary intervention (PCI)-is limited, mainly confined to a subanalysis of the North American BARI trial (1988-1991), suggesting a mortality benefit of CABG over PCI. No prospective trial has addressed this issue specifically.

Objective: The CARDia trial is an investigator initiated study and is the first prospective study designed to address the hypothesis that optimal PCI defined as stenting with abciximab is not inferior to up-to-date CABG as a revascularisation strategy for diabetics with multivessel or complex single vessel coronary disease. The primary end point is the well established composite of death, non-fatal myocardial infarction (MI), and cerebrovascular accident (CVA) at one year.

Method: A total of 600 patients with diabetes are to be randomised to either PCI or CABG, with few protocol restrictions on operative techniques or use of new technology. A cardiac surgeon and a cardiologist must agree that a patient is suitablefor revascularisation by either technique before recruitment into the study. If a patient fulfils the angiographic criteria for entry into the study but is not suitable for randomisation they will enter the registry. In the surgical arm arterial revascularisation, off pump surgery, and minimally invasive techniques will be encouraged but ultimately left to the surgeon's discretion.

Recruitment: Twenty centres in the UK and Ireland have been or are being set up to recruit patients. Recruitment started last year and is due to complete in 2004 . Over $15 \%$ of patients have now been recruited. Preliminary results relating to six month follow up are expected in 2004. Data on cost effectiveness, quality of life, and neurocognitive function are being collected.

Drug eluting stents in CARDia: A major limitation of PCI has always been in-stent restenosis and a subsequent requirement for further revascularisation procedures. The RAVEL trial suggested that the use of rapamycin coated stents during PCI can reduce or even prevent restenosis, but very few of these patients were diabetic. The SIRIUS trial, recently presented in Washington, shows that while patients with diabetes, who received rapamycin coated stents, still have an increased requirement for target lesion revascularisation compared to non-diabetics, this was reduced by two thirds compared to patients with diabetes who received bare stenting.

In 2002 rapamycin coated stents became available and patients in the PCI arm are being further randomised between bare and coated stents. It has been calculated that this will have little impact on the primary end point of death, MI, and CVA but will allow a comparison of death, MI, and target lesion revascularisation between the bare stents and coated stents in the PCI arm.

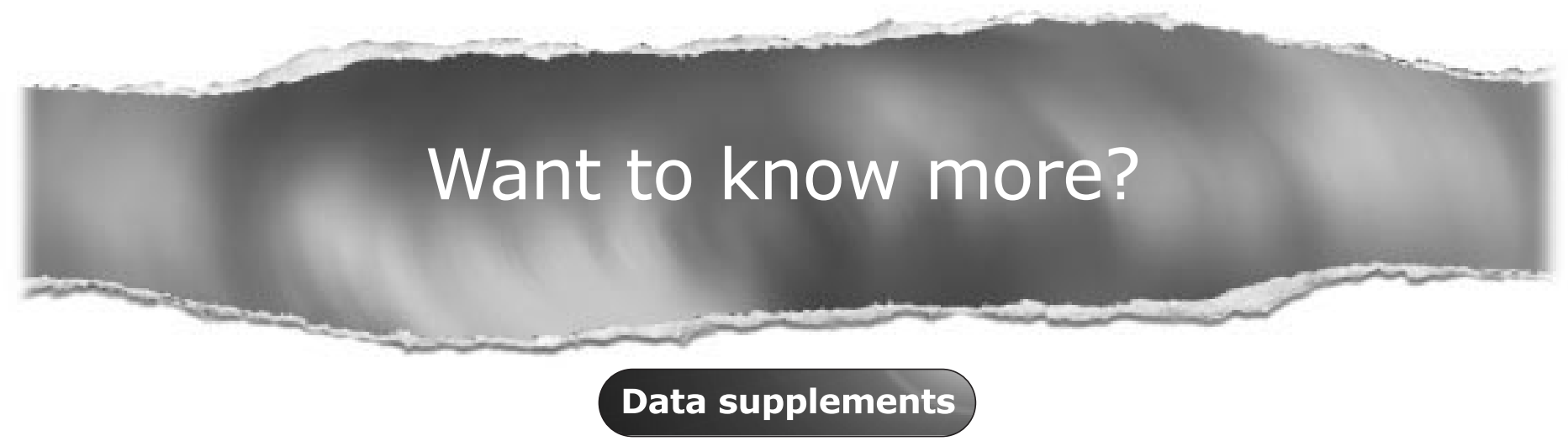

Limited space in printed journals means that interesting data and other material are often edited out of articles; however, limitless cyberspace means that we can include this information online. Look out for additional tables, references, illustrations, and (soon to appear) multimedia clips online! 